\title{
DESARROLLO Y PRUEBA PILOTO PSICOMÉTRICA DE UN CUESTIONARIO PARA LA EVALUACIÓN DE LA SOBRECARGA DEL CUIDADOR DURANTE LA ATENCIÓN DOMICILIARIA EN ENFERMERÍA
}

\author{
DEVELOPMENT AND PSYCHOMETRIC PILOT TEST OF A \\ QUESTIONNAIRE FOR THE EVALUATION OF CAREGIVER \\ OVERLOAD DURING HOME CARE IN NURSING
}

Sonia Ayuso-Margañón (1) , Núria Rodríguez-Ávila (2), M Carme Riera-iPrunera (3)

(1) Instituto Catalán de la Salud y Departamento de Enfermería de Salud Pública, Salud Mental y Materno-infantil, Facultad de Medicina y Ciencias de la Salud, Universidad de Barcelona.

Feixa Llarga s/n, 08907 L'Hospitalet de Llobregat. Barcelona. España. https://orcid.org/ 0000-0002-0105-6300

(2) Departamento de Sociología, Universidad de Barcelona. Avenida Diagonal 690, 08034 Barcelona. España. https://orcid.org/0000-0001-9746-2495

(3) Departamento de Econometría, Estadística y Economía Aplicada, Universidad de Barcelona. Avenida Diagonal 690, 08034 Barcelona. España. https://orcid.org/ 0000-0001-5784-2231

Ayuso Margañón, S., Rodríguez Ávila, N., \& Riera i Prunera, M. (2020). Desarrollo y prueba piloto psicométrica de un cuestionario para la evaluación de la sobrecarga del cuidador durante la atención domiciliaria en enfermería. Revista Ene de Enfermería, 14(1). Consultado de http://ene-enfermeria.org/ojs/index.php/ENE/article/view/977 


\section{Resumen}

El objetivo de este estudio fue evaluar las propiedades psicométricas del Cuestionario de Evaluación de la sobrecarga del cuidador (CESC) en la atención domiciliaria de enfermería.

El estudio responde a una modalidad de investigación empírico-descriptiva y el diseño atiende a una metodología de tipo mixta. El estudio se desarrolló en 4 fases: marco teórico, diseño del cuestionario, prueba piloto (la muestra de estudio la componían 14 enfermeras/os de tres Centros de Atención Primaria del área de L'Hospitalet de Llobregat y 296 cuidadores; las variables de análisis fueron los síntomas y predictores de sobrecarga asociados al cuidador y al paciente) y medición de las características psicométricas.

Se ha diseñado una escala que es estadísticamente fiable (KR-20 $=0,72)$, consistente y válida $(r=0,79 ; \mathrm{KMO}=0,74)$ y que permite medir la sobrecarga del cuidador con mayor precisión.

Palabras clave: Cuidadores, enfermería de salud en el domicilio, cuidados de enfermería, cuestionario.

\section{Abstract}

The aim of this study was to evaluate the psychometric properties of the Caregiver Overload Assessment Questionnaire (CESC) in home nursing care.

This study responds to an empirical-descriptive research modality and the design follows a mixed type methodology. The study was developed in 4 phases: theoretical framework, design of the questionnaire, pilot test (the study sample consisted of 14 nurses from three Primary Care Centres in the area of L'Hospitalet de Llobregat and 296 carers; the variables under analysis were the symptoms and overload predictors associated with the caregiver and patient) and measurement of psychometric characteristics.

A scale that is statistically reliable (KR-20 $=0.72)$, consistent and valid $(r=0.79 ; \mathrm{KMO}=0.74)$ has been designed to measure caregiver overload more accurately.

Keywords: Caregivers, home health nursing, nursing care, questionnaire. 


\section{INTRODUCCIÓN}

Actualmente, el instrumento que se utiliza de forma habitual para la evaluación del grado de sobrecarga que experimentan los cuidadores es la adaptación del Zarit Burden Interview, conocido comúnmente como Escala de Zarit (1). Concretamente, la versión española del cuestionario, realizada por Martín et al. (2) en 1996, en cuyo anexo 1 se presenta el cuestionario traducido, pero con una escala de frecuencia codificada de 1 a 5.

El Zarit se basa en la evaluación subjetiva que realiza el cuidador, no teniendo en cuenta el conjunto de factores predictores y síntomas que intervienen en la sobrecarga, los cuales son valorados durante la atención domiciliaria por las enfermeras/os, como profesionales que abarcan gran parte de las intervenciones de soporte de los cuidadores informales (1). A esto se añade el inconveniente de su extensión y, por tanto, el tiempo que se emplea en su uso.

En estas circunstancias se justifica la existencia de un nuevo cuestionario para evaluar la sobrecarga del cuidador que satisfaga las necesidades de las enfermeras/os, pero manteniendo los mismos criterios de validez y/o fiabilidad que el Zarit, que ha demostrado su utilidad en otros estudios.
En la literatura se han enumerado un conjunto de variables relacionadas de forma significativa con la sobrecarga. Los estudios hablan de un nivel elevado de dependencia física como uno de los principales factores predictores de malestar en los cuidadores, junto con el deterioro cognitivo del paciente $(3,4)$. Sin embargo, los responsables de una parte importante de la sobrecarga familiar son los factores conductuales que conlleva una demencia $(5,6)$. Además, la convivencia continua con la persona dependiente incrementa la percepción subjetiva de sobrecarga (7) y el impacto negativo sobre la salud del hecho de cuidar se percibe con doble frecuencia entre las mujeres (8).

Del mismo modo, diversos autores coinciden en que los cónyuges cuidadores son los que experimentan el mayor nivel de sobrecarga, y que un nivel socioeconómico bajo en cuidadores se relaciona con una mayor percepción de sobrecarga del cuidador y un peor ajuste y bienestar psicológico $(9,10)$. Por otro lado, el afrontamiento racional del cuidador y el apoyo proporcionado por la familia, los amigos o los recursos sociales y, sobre todo, la satisfacción que perciban los cuidadores con el apoyo recibido aliviará la sobrecarga percibida por el cuidador $(11,12)$. 
Por último, el cuidado permanente de una persona dependiente puede desencadenar consecuencias, tanto a nivel físico como psicosocial, en la persona que está realizando dichos cuidados. Entre las alteraciones físicas se encuentran la fatiga, debilidad muscular, dispepsia, vértigo, cefaleas, insomnio, dolores osteoarticulares, palpitaciones, trastornos de memoria, falta de concentración e inapetencia, de los cuales la literatura destaca los problemas de sueño y la fatiga como los aspectos físicos más notables en la sobrecarga del cuidador $(13,14)$. En relación a las alteraciones psicosociales podemos clasificarlas en tres grupos según las reacciones que desencadenan en la experiencia afectiva, social o comportamental, respectivamente (15): síntomas emocionales (ansiedad, depresión, ira, irritabilidad, baja autoestima y desesperanza), síntomas sociales (demanda de ayuda, situaciones conflictivas y aislamiento social) y síntomas reflejados en comportamientos negativos (distanciamiento $\mathrm{o}$ descuido del paciente, preocupación excesiva por el paciente, no cuidar sus propias necesidades y fumar más de lo normal, beber o comer más). De estas alteraciones destacan ansiedad y depresión como los principales problemas manifiestos en los cuidadores con sobrecarga $(16,17)$.
A raíz de ello, el objetivo de este estudio fue evaluar las propiedades psicométricas del Cuestionario de Evaluación de la sobrecarga del cuidador (CESC). Se trata de un test más sencillo y reducido, que fue diseñado para responder a las necesidades actuales de paciente, cuidador y profesional sanitario en el marco de la atención domiciliaria (18).

\section{MATERIAL Y MÉTODO}

El estudio responde a una modalidad de investigación empírico-descriptiva, y el diseño utilizado atiende a una metodología mixta, combinando una perspectiva cualitativa y cuantitativa.

El estudio se ha desarrollado en 4 fases: I. Marco teórico; II. Planificación y diseño del instrumento; III. Prueba piloto y IV. Medición de las características psicométricas. En este artículo nos centraremos en la fase III y la IV.

En la primera fase, se llevó a cabo la búsqueda de información y la recogida de datos a través de una revisión bibliográfica y 18 entrevistas semiestructuradas elaboradas ad hoc a cuidadores y profesionales sanitarios como enfermeras/os, gestoras de casos y trabajadoras sociales (19).

En la fase II del estudio, la triangulación y la categorización de los datos 
procedentes de las diversas fuentes permitió diseñar el instrumento de valoración de la sobrecarga del cuidador $(20,21)$. El proceso de codificación y análisis de los datos cualitativos se llevó a cabo mediante el software Atlas-ti. El instrumento, conocido como Cuestionario de Evaluación de la sobrecarga del cuidador (CESC), mide a través de 16 ítems varios aspectos de la sobrecarga que son fácilmente obtenidos a partir de la valoración enfermera. Estos aspectos son síntomas y factores predictores de sobrecarga asociados al cuidador y al paciente, y constituyen variables que miden la carga objetiva y subjetiva. En esta fase, el CESC se sometió a una validación de contenido, mediante el método de Agregados Individuales, de manera que 3 expertos recogieron y analizaron los ítems del instrumento para juzgar cuáles podían quedar incluidos y los que debían ser excluidos hasta decidir una versión consensuada $(18,22)$.

Posteriormente, en la fase III, se efectuó una prueba piloto durante un período de 6 meses, en la cual se puso en marcha la propuesta consensuada en la etapa anterior. La muestra de estudio la formaron 14 enfermeras/os de tres Centros de Atención Primaria del área de L'Hospitalet de Llobregat (Barcelona) y 296 cuidadores informales de población adulta, durante el período comprendido entre el 1 de enero de 2016 y el 30 de noviembre de 2017. La captación de cuidadores y recogida de datos fue llevada a cabo por las enfermeras/os. La técnica de muestreo seleccionada fue el muestreo no probabilístico por conveniencia. Las enfermeras/os y cuidadores fueron seleccionados en base a los criterios de inclusión establecidos. El trabajo de campo requirió un registro de la valoración y del seguimiento del cuidador por parte de las enfermeras/os participantes, utilizando la herramienta diseñada.

Finalmente, en la fase IV, con los resultados obtenidos, se llevó a cabo el proceso de análisis de los datos y medición de las características psicométricas del instrumento para determinar su factibilidad, validez y fiabilidad, utilizando el programa estadístico informático SPSS v.24 (23).

La factibilidad se evaluó por medio de una encuesta de opinión y satisfacción administrada a las enfermeras/ os; constaba de 10 reactivos divididos en 3 bloques: reactivos relacionados con el contenido, relacionados con el diseño y relacionados con la práctica clínica. Se analizó la validez de criterio, estableciendo la correlación entre el resultado obtenido en el nuevo instrumento diseñado y el considerado como patrón de referencia, el cuestionario de Zarit, mediante el cálculo del coeficiente de corre- 
AYUSO-MARgAÑón - RODRÍGUEZ-ÁVILA - RIERA I PRUNERA CUESTIONARIO PARA LA EVALUACIÓN DE LA SOBRECARGA DEL CUIDADOR

lación de Pearson y la representación gráfica (24). Para analizar la fiabilidad se utilizó el método de Consistencia Interna a través de la fórmula 20 de Kuder-Richardson (KR-20). La validez del constructo se estudió a través del análisis factorial; se empleó el método de Componentes Principales con rotación Varimax y prueba de sedimentación de Cattell para la extracción de los factores, y se retuvieron aquellos factores cuyos autovalores fueran superiores a 1,00. En relación a los ítems, se consideró 0,40 como el peso mínimo para determinar la asignación de los ítems a los factores (25). De este modo, se obtuvo la versión definitiva (figura 1).

\section{CUESTIONARIO DE EVALUACIÓN DE LA SOBRECARGa DEL CUIDADOR ${ }^{1}$}

Responder a las preguntas de los dos bloques (cuidador y paciente) marcando la casilla correspondiente, según la percepción y/o conocimiento del profesional. Los valores 0-4 representan la ponderación de cada respuesta. Para obtener la puntuación final, debe sumar el valor de sus respuestas según lo que indique cada columna.

\section{CUIDADOR}

Marque la casilla correspondiente a los siguientes datos y síntomas relacionados con el cuidador.

\begin{tabular}{|c|c|c|c|c|c|}
\hline Puntuación: & 0 & 1 & 2 & 3 & 4 \\
\hline Género & ○ Hombre & $\begin{array}{l}\circ \\
\text { Mujer }\end{array}$ & & & \\
\hline Parentesco & - Otros & $\begin{array}{l}\circ \\
\text { Parej } \\
\text { a }\end{array}$ & & & \\
\hline Nivel de ingresos & $\begin{array}{l}\text { Medio o } \\
\text { Alto }\end{array}$ & - Bajo & & & \\
\hline $\begin{array}{l}\text { ¿Convive en el mismo domicilio con el } \\
\text { paciente? }\end{array}$ & $\circ$ No & o Sí & & & \\
\hline $\begin{array}{l}\text { ¿Carece de soporte social/familiar? } \\
\text { (Ayudas a domicilio, prestaciones económicas, } \\
\text { ingresos por descanso familiar, Telealarma, } \\
\text { cuidador remunerado, otros familiares...) }\end{array}$ & $\circ$ No & & & - Sí & \\
\hline $\begin{array}{l}\text { ¿Piensa el cuidador que la ayuda recibida es } \\
\text { insuficiente o inadecuada? } \\
\text { (responder SÓLO si dispone de soporte social/ } \\
\text { familiar) }\end{array}$ & $\circ$ No & & & & - Sí \\
\hline
\end{tabular}

\footnotetext{
1 Este cuestionario debe ser completado por el profesional sanitario de referencia con conocimiento del paciente y de su cuidador.
} 
AYUSO-MARgAÑón - RODRÍGUEZ-ÁVILA - RIERA I PRUNERA CUESTIONARIO PARA LA EVALUACIÓN DE LA SOBRECARGA DEL CUIDADOR

\begin{tabular}{|c|c|c|c|c|c|}
\hline $\begin{array}{l}\text { Síntomas emocionales (puede presentar uno } \\
\text { o varios): } \\
\text { Ansiedad (nerviosismo, angustia, tensión, } \\
\text { estrés); depresión (llanto, tristeza, apatía); } \\
\text { desesperanza (reconoce que "no puede más") }\end{array}$ & $\circ$ No & & & & ○ Sí \\
\hline $\begin{array}{l}\text { Síntomas sociales (puede presentar uno o } \\
\text { varios): } \\
\text { Múltiples demandas de ayuda (visitas o } \\
\text { contactos repetidos al CAP); aislamiento social }\end{array}$ & $\circ$ No & & & ○ Sí & \\
\hline $\begin{array}{l}\text { Comportamientos negativos (puede presentar } \\
\text { uno o varios): } \\
\text { Preocupación excesiva por el paciente; descuido } \\
\text { del paciente; descuido de las propias } \\
\text { necesidades }\end{array}$ & $\circ$ No & & & ○ Sí & \\
\hline $\begin{array}{l}\text { Síntomas físicos (puede presentar uno o } \\
\text { varios): } \\
\text { Cansancio (falta de fuerzas, fatiga); dificultad } \\
\text { para dormir }\end{array}$ & $\circ$ No & & ○ Sí & & \\
\hline $\begin{array}{l}\text { Síntomas físicos (puede presentar uno o } \\
\text { varios): } \\
\text { Palpitaciones; trastornos de memoria }\end{array}$ & $\circ$ No & ○ Sí & & & \\
\hline $\begin{array}{l}\text { Afrontamiento/actitud del cuidador frente al } \\
\text { problema: }\end{array}$ & - Positiva & & & & - Negativa \\
\hline $\begin{array}{r}\text { (sume la puntuación total según el valor de cada } \\
\text { columna) }\end{array}$ & & & & & Subtotal: \\
\hline
\end{tabular}

\section{PACIENTE}

Marque la casilla correspondiente a los siguientes datos relacionados con el paciente:

\begin{tabular}{|c|c|c|c|c|c|}
\hline Puntuación: & 0 & 1 & 2 & 3 & 4 \\
\hline ¿Es un paciente en fase final de vida?/MACA & $\circ$ No & & ○ Sí & & \\
\hline $\begin{array}{l}\text { Nivel de dependencia severa: } \\
\text { Barthel }(\leq 35) \text { o Lawton y Brody }(\leq 3 \text { 우 } / \leq 1 \hat{\delta})\end{array}$ & $\circ$ No & & & & ○ Sí \\
\hline $\begin{array}{l}\text { Pfeiffer } \geq 5 \text { errores o diagnóstico de demencia } \\
\text { (Deterioro cognitivo moderado a importante) }\end{array}$ & $\circ$ No & & & ○ Sí & \\
\hline $\begin{array}{l}\text { Presencia de trastornos conductuales o del } \\
\text { comportamiento: } \\
\text { (Agitación, insomnio, agresividad, hiperactividad, } \\
\text { delirios, gritos...) }\end{array}$ & $\circ$ No & & & & ○ Sí \\
\hline \multirow[t]{2}{*}{$\begin{array}{r}\text { (sume la puntuación total según el valor de cada } \\
\text { columna) }\end{array}$} & \multicolumn{5}{|r|}{$\begin{array}{r}\text { Subtotal } \\
\text { : }\end{array}$} \\
\hline & & \multicolumn{4}{|c|}{$\begin{array}{l}<5 \text { puntos_No se observa sobrecarga } \\
5-11 \text { puntos_Sobrecarga leve } \\
12-19 \text { puntos_Sobrecarga intensa } \\
\geq 20 \text { puntos_Sobrecarga muy intensa }\end{array}$} \\
\hline
\end{tabular}


Figura 1. Versión definitiva del Cuestionario de Evaluación de Sobrecarga del Cuidador (CESC)

Respecto a los aspectos éticos, los participantes fueron informados adecuadamente sobre la investigación y todos los aspectos que conllevaba su colaboración. El acceso a la Historia clínica de los pacientes se llevó a cabo bajo secreto profesional desde el Centro de Atención Primaria (CAP). El estudio se llevó a cabo en consonancia con el código ético de la Organización Mundial de la Salud (Declaración de Helsinki) y ha sido sometido a la valoración y aprobación del Comité de Bioética de la Universidad de Barcelona obteniendo un informe favorable.

\section{REsultados}

Como se especifica en el apartado anterior, también nos hemos centrado en los resultados obtenidos en la fase III y la IV. En primer lugar, un análisis de frecuencias permitió conocer las características de las personas estudiadas. Más de la mitad de la muestra estudiada eran mujeres (68\%); solo un 34\% eran cónyuges; casi la mitad de los cuidadores presentaban un nivel bajo de ingresos (42\%); la mayor parte de ellos (70\%) convivían en el mismo domicilio con el paciente al que cuidaban y casi toda la muestra (82\%) disponía de soporte social para el cuidado o bien de soporte de otros familiares; el $51 \%$ de los cuidadores de la muestra sufren síntomas emocionales relacionados con la ansiedad y desesperanza; solo un $24 \%$ presentan síntomas sociales, tales como las múltiples demandas de ayuda o aislamiento social; una cuarta parte de los cuidadores presenta algún comportamiento negativo, como preocupación excesiva por el paciente, descuido del mismo o de las propias necesidades; más de la mitad del total de cuidadores (57\%) presentan los síntomas de cansancio e insomnio; hay un porcentaje muy bajo (19\%) de síntomas como palpitaciones y trastornos de memoria; el $80 \%$ de los presentan una actitud positiva frente al problema. Por último, respecto a los pacientes, el $54 \%$ presentaba un nivel de dependencia severo, teniendo el $20 \%$ de ellos un pronóstico de vida limitado; el 35\% estaban diagnosticados de demencia 0 sufrían un deterioro cognitivo de moderado a severo, y un $23 \%$ sufrían trastornos conductuales o del comportamiento.

Las pruebas de factibilidad del CESC fueron favorables con un prome- 
dio de 4,46 (mediana, 4,55 y moda 5). El elemento con un mayor grado de satisfacción para el grupo (promedio de 4,67) fue el reactivo 5 valorado por 8 de los 12 participantes con la máxima puntuación, obteniendo, además, un alto grado de homogeneidad en el grupo (Coeficiente de variación $=0,11)$. Hubo una valoración más moderada en el último bloque (reactivos 8-10) en que se incluían los reactivos referentes a la práctica clínica (tabla 1). En cuanto a la opinión y los comentarios del grupo, las principales aportaciones comentadas sobre el CESC hacían referencia a su facilidad de uso y adecuación a las necesidades del paciente y del profesional que realizaba la valoración.

Tabla 1. Resumen de resultados referente al grado de satisfacción sobre el CESC.

Reactivos

1. La información del cuestionario resulta clara y entendedora.

2. El cuestionario incluye la información conveniente sobre el cuidador y el paciente.

3. La información del cuestionario resulta adecuada para valorar la sobrecarga del cuidador.

4. El formato resulta ameno.

5. El cuestionario se cumplimenta de forma fácil.

6. El tiempo dedicado a la cumplimentación del cuestionario es apropiado.

7. La codificación de los resultados del 0 al 4 resulta sencilla.

8. Permite identificar elementos que pueden influir negativa o positivamente en el cuidador para priorizar áreas de intervención.

9. Para la evaluación de la sobrecarga del cuidador preferiría el test propuesto a otros similares.

10. Recomendaría este instrumento a otros profesionales.

Total

Mínimo

\begin{tabular}{|c|c|c|c|c|}
\hline Media & Mediana & Moda & $\mathrm{DE}$ & $\mathrm{CV}$ \\
\hline 4,58 & 5,0 & 5 & 0,51 & 0,11 \\
\hline 4,50 & 4,5 & 5 & 0,52 & 0,12 \\
\hline 4,58 & 5,0 & 5 & 0,51 & 0,11 \\
\hline 4,50 & 4,5 & 4 & 0,52 & 0,12 \\
\hline 4,67 & 5,0 & 5 & 0,49 & 0,11 \\
\hline 4,50 & 4,5 & 4 & 0,52 & 0,12 \\
\hline 4,33 & 4,0 & 4 & 0,65 & 0,15 \\
\hline 4,33 & 4,5 & 5 & 0,78 & 0,18 \\
\hline 4,42 & 4,5 & 5 & 0,67 & 0,15 \\
\hline 4,42 & 4,5 & 5 & 0,67 & 0,15 \\
\hline 4,33 & 4,0 & 4 & 0,49 & 0,11 \\
\hline 4,67 & 5,0 & 5 & 0,78 & 0,18 \\
\hline
\end{tabular}

DE: Desviación estándar; CV: Coeficiente de variación

Por lo que respecta a la validez de criterio de este estudio, ambos test presentan una relación lineal positiva; es decir, a medida que aumenta el valor del test de
Zarit, aumenta también el valor del nuevo cuestionario (figura 2). 


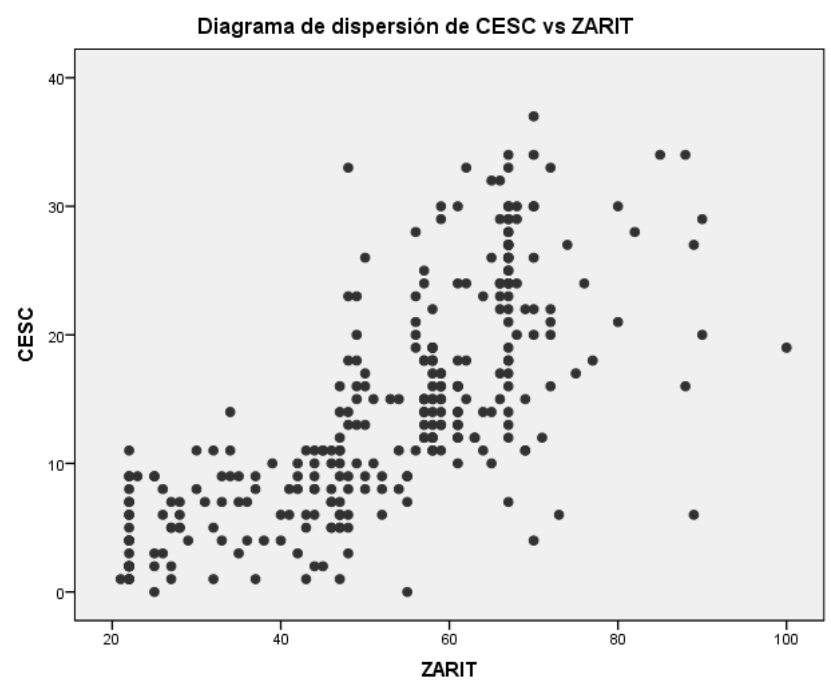

Figura 2. Diagrama de dispersión de los resultados del CESC vs ZARIT, en pacientes domiciliarios de Atención Primaria, L'Hospitalet de Llobregat, Barcelona, 2017

Cabe mencionarse que, para el cálculo de la fórmula de Pearson, fue necesario transformar las puntuaciones en rangos. En cuanto al test de Zarit se seleccionaron los valores en función de su propio rango de respuestas, de manera que las puntuaciones inferiores a 46 ("no sobrecarga") adquirieron el valor 1, las puntuaciones entre 46 y 56 ("sobrecarga leve") adoptaron el valor 2 y las puntuaciones superiores a 56 ("sobrecarga intensa") tomaron el valor 3 . Por otro lado, en el CESC se decidió incluir un nivel de sobrecarga adicional ("sobrecarga moderada"), puesto que se consideró que debía haber un nivel de detalle mayor entre el nivel de "sobrecarga leve" y el de "intensa". Sin embargo, para verificar si existía una posible correspondencia del CESC con el test de Zarit, se tu- vieron que establecer también tres rangos, por lo que fue necesario fusionar el rango de "sobrecarga moderada" con uno de los dos niveles colaterales ("sobrecarga leve" o "sobrecarga intensa"). La decisión final vino determinada por el grado de correlación que se obtuvo realizando el análisis con ambas posibilidades. Se aplicaron a la misma muestra los dos instrumentos de evaluación y el CESC correlacionó 0,58 con las puntuaciones en el primer caso y 0,79 con las correspondientes puntuaciones en el segundo caso. Dado que la fusión con el nivel de "sobrecarga intensa" es la que ofreció mejores resultados de correlación con el test de Zarit, se procedió a tomar la decisión de renombrar las cuatro categorías de sobrecarga del CESC. El valor de correlación de Pearson fue de 0,79; lo 
cual permite hablar de una correlación considerable (26).

En lo que respecta a la fiabilidad, el análisis de los ítems aplicando el cálculo del KR-20 al CESC reveló un nivel aceptable de confiabilidad con los 16 ítems del instrumento, obteniendo un valor de 0,72 (correlación positiva).

En cuanto a la validez del constructo, se comprobó que la matriz de los datos era adecuada para el análisis factorial. La prueba de Kaiser-Meyer-Olkin $(\mathrm{KMO}=0,744)$ es claramente superior a 0,5 , lo cual permite contrastar la validez de acuerdo con lo establecido (27). Asimismo, la prueba de esfericidad de Bartlett arrojó un valor significativo $\left(\mathrm{X}^{2}\right.$ $(105)=857.233 ; p=0.000)$. Al ser inferior a $p<0.05$ indica que el análisis factorial es adecuado, ya que se considera que las variables están los suficientemente interrelacionadas para realizarlo. En el análisis de la diagonal de la matriz anti-imagen se puede ver el valor de las medidas de adecuación que presenta cada variable para el cálculo de los factores, y que se conoce como "Measure of Sampling Adecuacy" (MSA) (su rango varía entre 0 y 1 ). Se observó que los ítems presentaron buenos niveles de adecuación con valores en general supe- riores a 0,60 , siendo éste un indicador positivo de la matriz de datos. La variable con un nivel de adecuación menor fue el "Género" ( $r=0,523)$. No obstante, es preciso considerar que la base muestral de la prueba piloto era mayoritariamente femenina existiendo posiblemente un sesgo por contexto histórico y cultural.

A partir de aquí se procedió a la extracción de los distintos factores a través de la agrupación de 15 variables. El análisis factorial reveló 5 factores y la solución explicó el 58,99\% de la varianza. La regla de Kaiser-Guttman o regla K1 permitió identificar estos cinco factores con raíces latentes o autovalores mayores que 1. La solución forzando tres y cuatro factores no se encontró adecuada por dos razones: en primer lugar, explicaba solo el $44,48 \%$ y $52,18 \%$ de la varianza total respectivamente; en segundo lugar, tras analizar la matriz de factorización se observó que los ítems no guardaban coherencia teórica con el factor en el cual saturaban, observando ítems con pesajes mucho más bajos. A partir de la prueba de sedimentación de Cattell se observa un claro punto de inflexión que permitió conservar cinco factores (figura 3). 


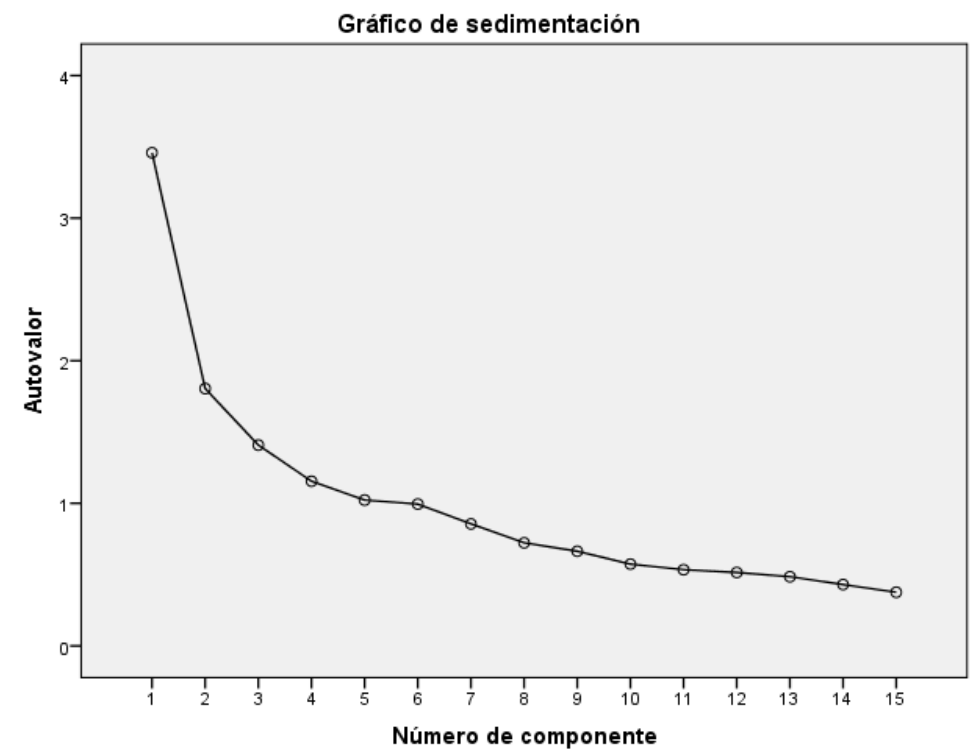

Figura 3. Gráfico de sedimentación para los ítems del CESC

En concreto, el factor 1, "expre- deterioro cognitivo; el factor 4, "red de sión emocional", incluye aspectos psicoapoyo", se refiere a recursos de soporte lógicos, el factor 2, "calidad vida", se resocial y familiar; por último, el factor 5 , fiere a problemas físicos así como a as"desarrollo social", incluye los aspectos pectos convivenciales; el factor 3 , "detesocioeconómicos (tabla 2).

rioro funcional y cognitivo", describe el nivel de dependencia del paciente y su

Tabla 2. Estructura factorial del cuestionario CESC en pacientes domiciliarios de Atención Primaria, L'Hospitalet de Llobregat, Barcelona, 2017 
Matriz de componente rotado ${ }^{a}$

\begin{tabular}{|c|c|c|c|c|c|}
\hline & \multicolumn{5}{|c|}{ Componente } \\
\hline & 1 & 2 & 3 & 4 & 5 \\
\hline Comportamientos negativos & 0,739 & & & & \\
\hline $\begin{array}{l}\text { Síntomas físicos (palpitaciones, trastornos de } \\
\text { memoria) }\end{array}$ & 0,665 & & & & \\
\hline Afrontamiento & 0,587 & & & & \\
\hline Síntomas emocionales & 0,570 & & & & \\
\hline Parentesco & & 0,792 & & & \\
\hline Convivencia & & 0,639 & & & \\
\hline Severidad de la patología & & 0,638 & & & \\
\hline Síntomas físicos (cansancio, dificultad para dormir) & & 0,485 & & & \\
\hline Deterioro cognitivo o demencia & & & 0,814 & & \\
\hline Dependencia & & & 0,763 & & \\
\hline Trastornos conductuales & & & 0,505 & & \\
\hline Percepción del soporte recibido & & & & 0,747 & \\
\hline Soporte social/familiar & & & & 0,743 & \\
\hline Síntomas sociales & & & & & 0,673 \\
\hline Nivel de ingresos & & & & & 0,625 \\
\hline
\end{tabular}

Método de extracción: análisis de componentes principales Método de rotación: Varimax con normalización Kaiser a.

a. La rotación ha convergido en 7 iteraciones

\section{DISCUSIÓN}

Los resultados hallados permiten afirmar que se ha diseñado un instrumento que demuestra ser estadística y conceptualmente fiable, consistente y válido para medir la sobrecarga del cuidador. En términos generales, los especialistas opinan que el CESC valora la sobrecarga del cuidador de una forma más alta. Este instrumento no solo está correlacionado con el test de Zarit, sino que proporciona más información y ma- yor detalle en el grado de sobrecarga. Respecto a la validación de criterio, se ha obtenido una correlación alta entre el CESC y el test de Zarit. Al determinar la validez de constructo también se han encontrado resultados satisfactorios. Los datos que ha aportado el análisis factorial muestran la posibilidad de considerar una estructura en la que los ítems del CESC están explicados en cinco dimensiones, los cinco factores emergentes tienen sentido psicológico y son de sen- 
cilla interpretación. En definitiva, es posible afirmar que el constructo es válido, en tanto que tiene una estructura que permite explicar una varianza significativa presente en las variables originales y examinar el significado de lo que estamos midiendo (28). La preeminencia del factor emocional expresa el gran impacto que representa la experiencia interna del cuidador en la sobrecarga, lo cual es consecuente con la bibliografía estudiada respecto a que las estrategias de afrontamiento que utiliza el cuidador condicionan la manera de adaptarse a la situación de cuidar al paciente y a él mismo (29). A continuación, aparecen los factores relacionados con la calidad de vida, ya que la situación de estrés que viven los cuidadores afecta a su estado de salud y bienestar. Por otro lado, destacan las características del paciente dependiente, lo cual ratifica la necesidad de incluir en la valoración de la sobrecarga aspectos del paciente y no solo del cuidador. Finalmente, están los recursos socioeconómicos, cuyo déficit confiere un grado más de vulnerabilidad al cuidador, lo que evidencia que sería posible minimizar la carga si existiese una red de apoyo social y familiar sólida (30). En definitiva, vemos que la sobrecarga psicoafectiva se impone por encima de la física y la social, por lo que sería importante priorizar las intervenciones de apo- yo emocional al cuidador para evitar la sobrecarga. EI CESC también evidenció valores aceptables de fiabilidad, de manera que se puede afirmar que los resultados del instrumento son consistentes y éste mide con precisión aquello que quiere medir. Asimismo, las pruebas de factibilidad destacan que los profesionales que han utilizado la nueva herramienta están altamente satisfechos con su mayor comodidad.

En el futuro se podría mejorar esta herramienta, aumentando la muestra y fijándonos en nuevos factores que puedan ser investigados como posibles componentes de nuestro constructo. También, sería necesario conocer las características psicométricas de la herramienta en diferentes poblaciones, dado que el buen desempeño de esta escala en el grupo estudiado no garantiza el mismo comportamiento en otros grupos de características sociales y culturales distintas. Finalmente es preciso considerar posibles sesgos en el estudio, como son el tamaño muestral de la prueba piloto y la realización y análisis de las entrevistas por las enfermeras/os.

Es obvio que resulta necesario considerar un cambio en la evaluación de sobrecarga del cuidador en el marco de la atención domiciliaria, de manera que la valoración de enfermería, que incluye el conocimiento de predictores y 
síntomas de sobrecarga del cuidador,

puede constituir un instrumento óptimo de medida en la determinación del nivel de sobrecarga del cuidador $(31,32)$. En este sentido, el CESC se puede recomendar como útil y válido, principalmente en el contexto de atención primaria, para la detección de sobrecarga en cuidadores de pacientes dependientes y su eventual intervención.

\section{AGRADECIMIENTOS}

Nuestro más sincero agradecimiento a las personas que participaron en el estudio por su amable disposición y tiempo prestado, sin las cuales no habría sido posible la realización de este. 


\section{BiBLIOGRAFÍA}

1. Álvarez L, González AM, Muñoz P. El cuestionario de sobrecarga del cuidador de Zarit. Cómo administralo e interpretarlo. Gac Sanit [Internet]. 2008 [consultado 10 Nov 2015]; 22:618-19. Disponible en: http://www.scielosp.org/pdf/gs/v22n6/carta1.pdf

2. Martin M, Salvadó I, Nadal S, et al. Adaptación para nuestro medio de la Escala de Sobrecarga del Cuidador (Caregiver Burden Interview) de Zarit. Revista Geronto. 1996; 6:338-346.

3. Torres P, Ballesteros E, Sánchez PD, et al. Programas, intervenciones y redes de apoyo a los cuidadores informales en salud. Necesidad de convergencia entre los programas formales y el apoyo informal. Nursing [Internet]. 2008 [consultado 10 Nov 2015]; 26:56-61. Disponible en: https:// doi.org/10.1016/S0212-5382(08)70757-X

4. Flores GE, Rivas RE, Seguel PF. Nivel de sobrecarga en el desempeño del rol del cuidador familiar del adulto mayor con dependencia severa. Ciencia y enfermería [Internet]. 2012 [consultado 10 Nov 2015]; 18:29-41. Disponible en: http://dx.doi.org/ 10.4067/S0717-95532012000100004

5. Conde Sala J. Demencia y familiares cuidadores: Deterioro, sobrecarga y apoyo social. Tiempo [Internet]. 2005 [consultado 10 Nov 2015]; 17:112. Disponible en: https://www.researchgate.net/ publication/274072952_Demencias_y_familiares_cuidadores_deterioro_sobrecarga_y_apoyo_social

6. Antón S. Cansancio del rol de cuidador: análisis de sus factores relacionados (I). Reduca (Enfermería, Fisioterapia Y Podología) [Internet]. 2014 [consultado 10 Nov 2015]; 6(3):74-106. Disponible en: http://www.revistareduca.es/index.php/reduca-enfermeria/article/viewFile/1708/1724

7. Velázquez Y, Espín AM. Repercusión psicosocial y carga en el cuidador informal de personas con insuficiencia renal crónica terminal. Rev Cubana Salud Pública [Internet]. 2014 [consultado 10 Nov 2015] 40(1), 3-17. Disponible en: http:// www.bvs.sld.cu/revistas/spu/vol40_1_14/ spu02114.htm

8. Larrañaga I, Martin U, Bacigalupe A, et al. Impacto del cuidado informal en la salud y la calidad de vida de las personas cuidadoras: análisis de las desigualdades de género. Gac Sanit [Internet]. 2008 [consultado 10 Nov 2015]; 22:443-50 https:// doi.org/10.1157/13126925

9. Kim H, Chang M, Rose K, et al. Predictors of caregiver burden in caregivers of individuals with dementia. J Adv Nurs [Internet]. 2012 [consultado 10 Nov 2015]; 68:846-55 https://doi.org/10.1111/j. 1365-2648.2011.05787.x

10.Noroozian I, Abdollahpour M, Nedjat S, et al. Caregiver Burden and its Determinants among the Family Members of Patients with Dementia in Iran.
International Journal of Preventive Medicine [Internet]. 2012 [consultado 10 Nov 2015]; 3(8):544551. Disponible en: http://www.ncbi.nlm.nih.gov/ pmc/articles/PMC3429801/

11.Crespo M, López J. El apoyo a los cuidadores de familiares mayores dependientes en el hogar: "Cómo mantener su bienestar" [Internet]. Madrid: Instituto de Mayores y Servicios Sociales (IMSERSO); 2007 [consultado 10 Nov 2015]. Disponible en: http://www.imserso.es/InterPresent1/groups/ imserso/documents/binario/apoyocuidadores.pdf

12.Fernández-Abascal EG. Estilos y estrategias de afrontamiento. Madrid: Pirámide; 1997.

13. Herrero MB. Factores de riesgo del cansancio del cuidador. Revista Enfermería CyL [Internet]. 2012 [consultado 10 Nov 2015]; 4:89-97. Disponible en:

14.http://www.revistaenfermeriacyl.com/index.php/ revistaenfermeriacyl/article/view/87/65

15.Barnosi A, Payán M, Sánchez $P$, et al. Descripción del grado de sobrecarga en el cuidador del enfermo con demencia en un hospital general. Revista Clínida Española [Internet]. 2014 [consultado 10 Nov 2015]; 214(460):6-7. Disponible en: http:// www.revclinesp.es/controladores/congresos-he$r \quad r \quad m$ i e $n$ t a $s$. $p \quad h \quad p$ ? idCongreso=15\&idSesion=1348\&idComunicacion= 14599

16.Piqueras JA, Ramos V, Martínez AE, et al. Emociones negativas y su impacto en la salud mental y física. Suma Psicológica [Internet]. 2009 [consultado 10 Nov 2015]; 16(2). Disponible en: http:// www.redalyc.org/pdf/1342/134213131007.pdf

17.López Gil MJ, Orueta R, Gómez Caro S, et al. El rol de Cuidador de personas dependientes y sus repercusiones sobre su calidad de vida y su salud. Revista Clínica de Medicina de Familia [Internet]. 2009 [consultado 10 Nov 2015]; 2:332-39. Disponible en: http://scielo.isciii.es/scielo.php? script =sci_arttext\&pid=S1699-695X2009000200004\&Ing=es

18.Dueñas E, Martínez MA, Morales B, et al. Síndrome del cuidador de adultos mayores discapacitados y sus implicaciones psicosociales. Revista Colombia Médica [Internet]. 2006 [consultado 10 Nov 2015]; 37(1):31-38. Disponible en: http:// www.bioline.org.br/pdf?rc06034

19. Ayuso S, Rodríguez N. Diseño y validación de contenido de un instrumento de evaluación de sobrecarga de la persona cuidadora de paciente dependiente. Metas de Enfermería [Internet]. 2018 [consultado Abr 2019]; 21(5):18-25. Disponible en: http://encuentra.enfermeria21.com/revistas/metas/ articulo/81242/

20.Ayuso S, Rodríguez N. Valoración de enfermería para la evaluación de sobrecarga del cuidador en atención domiciliaria. Horizonte Sanitario [Internet]. 2018 [consultado Abr 2019]; 17(3):179-187. Dispo- 
nible en: http://revistas.ujat.mx/index.php/horizonte/article/view/2147

21. Coffey A, Atkinson P. Encontrar el sentido a los datos cualitativos: estrategias complemetarias de investigación [Internet]. Antioquía (Turquía): Universidad de Antioquía; 2005 [consultado 2 Dic 2017]. Disponible en: http://www.fceia.unr.edu.ar/ geii/maestria/2014/DraSanjurjo/8mas/ Amanda\%20Coffey,\%20Encontar\%20el\%20sentido\%20a\%20los\%20datos\%20cualitativos.pdf

22. Rodriguez C, Pozo T, Gutierrez J. La triangulación analítica como recurso para la validación de estudios de encuesta recurrentes e investigaciones de réplica en Educación Superior. Revista Electrónica de Investigación y Evaluación Educativa [Internet]. 2006 [consultado 2 Dic 2017]; 12:289-305. Disponible en: https://www.uv.es/RELIEVE/v12n2/RELIEVEv12n2_6.htm

23. Corral Y. Validez y confiabilidad de los instrumentos de investigación para la recolección de datos. Ciencias de La Educación. [Internet]. 2009 [consultado 2 Dic 2017]; 19:229-47. Disponible en: http:// servicio.bc.uc.edu.ve/educacion/revista/n33/ art12.pdf

24.Carvajal A, Centeno C, Watson R, et al. How is an instrument for measuring health to be validated? An Sist Sanit Navar. [Internet]. 2011 [consultado 20 Feb 2018]; 34:63-72. Disponible en: http:// www.ncbi.nlm.nih.gov/pubmed/21532647

25. Elosua P. Sobre la validez de los tests. Psicothema. [Internet]. 2003 [consultado 20 Feb 2018]; 15:315-21. Disponible en: http://www.redalyc.org/ html/727/72715225/

26. Nunnally JC, Bernstein IH. Psychometric theory. $3^{\mathrm{a}}$ ed. Nueva York: Mc Graw-Hill; 1994. 752 p.

27.Martínez-Ortega RM, Tuya-Pendás L, MartínezOrtega M, et al. El coeficiente de correlación de los rangos de Spearman. Caracterización. Revista Habanera de Ciencias Médicas. [Internet]. 2009 [consultado 20 Feb 2018]; 8:1-12. Disponible en: http://scielo.sld.cu/scielo.php? script $=$ sci_arttext\&pid=S1729-519X2009000200017

28. Lloret Segura S. Ferreres Traver A, Hernández Baeza A, et al. El análisis factorial exploratorio de los ítems: una guía práctica, revisada y actualizada. Anales de Psicología. [Internet]. 2014 [consultado 20 Feb 2018]; 30:1151-69 https://dx.doi.org/ 10.6018/analesps.30.3.199361

29. Morales, P. El análisis factorial en la construcción e interpretación de tests, escalas y cuestionarios [Internet]. Madrid: Universidad Pontificia Comillas; 2013 [consultado 20 Feb 2018]. Disponible en: http://web.upcomillas.es/personal/peter/investigacion/AnalisisFactorial.pdf

30. Aldana G, Guarino L. Sobrecarga, afrontamiento y salud en cuidadoras de pacientes con demencia tipo Alzheimer. Revista Summa Psicológica UST.
[Internet]. 2012 [consultado 20 Feb 2018]; 9:5-14. Disponible en: https://dialnet.unirioja.es/servlet/ articulo?codigo $=3974398$

31.Flores E, Rivas E, Seguel F. Nivel de sobrecarga en el desempeño del rol del cuidador familiar de adulto mayorcon dependencia severa. Ciencia y Enfermería. [Internet]. 2012 [consultado $20 \mathrm{Feb}$ 2018];18:29-41 http://dx.doi.org/10.4067/ S0717-95532012000100004

32. Duque MC. Nurses Home : Enfermería en el domicilio. Un enfoque integrado de la Enfermería ante los retos del futuro. Revista de Administración Sanitaria Siglo XXI [Internet]. 2009 [consultado 20 Feb 2018]; 7(2):323-29. Disponible en: http:// www.elsevier.es/es-revista-revista-administracionsanitaria-siglo-xxi-261-articulo-nurses-home-enfermeria-el-domicilio--13139768

33.Rivera V, Pastoriza P, Díaz C, et al. Revisión crítica de la taxonomia enfermera NANDA internacional 2009-2011. Recien, Revista Electrónica Científica de Enfermería [Internet]. 2013 [consultado 20 Feb 2018]; 6:18. Disponible en: http://www.recien.scele.org/documentos/num_6_may_2013/ revis_critica_taxonomia_enfermera.pdf 